\title{
Myocardial perfusion MRI shows impaired perfusion of the mouse hypertrophic left ventricle
}

\author{
Bastiaan J. van Nierop • Bram F. Coolen • Noortje A. Bax • \\ Wouter J. R. Dijk · Elza D. van Deel - Dirk J. Duncker • \\ Klaas Nicolay • Gustav J. Strijkers
}

Received: 7 August 2013/ Accepted: 15 January 2014/Published online: 28 January 2014

(C) Springer Science+Business Media Dordrecht 2014

\begin{abstract}
There is growing consensus that myocardial perfusion deficits play a pivotal role in the transition from compensated to overt decompensated hypertrophy. The purpose of this study was to systematically study myocardial perfusion deficits in the highly relevant model of pressure overload induced hypertrophy and heart failure by transverse aortic constriction (TAC), which was not done thus far. Regional left ventricular (LV) myocardial perfusion $(\mathrm{mL} / \mathrm{min} / \mathrm{g})$ was assessed in healthy mice $(\mathrm{n}=6)$ and mice with TAC $(\mathrm{n}=14)$. A dual-bolus first-pass perfusion MRI technique was employed to longitudinally quantify myocardial perfusion values between 1 and 10 weeks after surgery. LV function and morphology were quantified from
\end{abstract}

Electronic supplementary material The online version of this article (doi:10.1007/s10554-014-0369-0) contains supplementary material, which is available to authorized users.

B. J. van Nierop · W. J. R. Dijk · K. Nicolay ·

G. J. Strijkers $(\square)$

Biomedical NMR, Department of Biomedical Engineering,

Eindhoven University of Technology, PO Box 513,

5600 MB Eindhoven, The Netherlands

e-mail: g.j.strijkers@tue.nl

B. F. Coolen

Department of Radiology, Academic Medical Center,

PO Box 22660, 1100 DD Amsterdam, The Netherlands

N. A. Bax

Soft Tissue Biomechanics and Engineering, Department of Biomedical Engineering, Eindhoven University of Technology, Eindhoven, The Netherlands

E. D. van Deel · D. J. Duncker

Experimental Cardiology, Thoraxcenter, Erasmus MC,

University Medical Center Rotterdam, PO Box 2040,

3000 CA Rotterdam, The Netherlands cinematographic MRI. Myocardial rest perfusion values in both groups did not change significantly over time, in line with the essentially constant global LV function and mass. Myocardial perfusion was significantly decreased in TAC mice $(4.2 \pm 0.9 \mathrm{~mL} / \mathrm{min} / \mathrm{g})$ in comparison to controls $(7.6 \pm 1.8 \mathrm{~mL} / \mathrm{min} / \mathrm{g})(P=0.001)$. No regional differences in perfusion were observed within the LV wall. Importantly, increased LV volumes and mass, and decreased ejection fraction correlated with decreased myocardial perfusion $(P<0.001$, in all cases). Total $\mathrm{LV}$ blood flow was decreased in TAC mice $(0.5 \pm 0.1 \mathrm{~mL} /$ min, $P<0.001)$ in comparison to control mice $(0.7 \pm 0.2 \mathrm{~mL} / \mathrm{min})$. Myocardial perfusion in TAC mice was significantly reduced as compared to healthy controls. Perfusion was proportional to LV volume and mass, and related to decreased LV ejection fraction. Furthermore, this study demonstrates the potential of quantitative first-pass contrast-enhanced MRI for the study of perfusion deficits in the diseased mouse heart.

Keywords MRI - First-pass perfusion - Mouse · Hypertrophy · Heart failure · Transverse aortic constriction

\section{Background}

Heart failure (HF) is a progressive syndrome in which the heart is no longer capable of pumping blood at a rate commensurate with the peripheral needs [1]. HF is an important cause of morbidity and mortality [2-4]. In many patients HF results from sustained, systemic hypertension resulting in chronic left ventricular (LV) pressure overload [5]. The heart initially adapts to this overload by means of hypertrophic growth. However, a broad range of concomitant maladaptive processes, including increased collagen 
deposition, metabolic changes and microvascular remodeling, eventually may lead to HF [6-8].

It has long been appreciated that pressure-overload induced LV hypertrophy is associated with alterations in the coronary microvasculature [9-12]. The pressure-overload hypertrophied LV is characterized by perivascular fibrosis and arterial medial hypertrophy, while vascular densities are lower as neovascularization does not occur commensurate with myocardial growth [13, 14]. These abnormalities force the myocardium to recruit its vasodilator reserve already under resting conditions, which occurs at the expense of its ability to increase myocardial blood flow in response to a stressor or vasodilator [15-17]. The resultant myocardial perfusion abnormalities during increased oxygen demands likely have a negative impact on cardiac function. However, the precise role of impaired perfusion in the development of HF remains incompletely understood. For example, it is unclear whether impaired perfusion is merely a consequence of hypertrophy or actually drives maladaptive remodeling.

Transverse aortic constriction (TAC) in mice is a highly relevant model of pressure overload induced hypertrophy and plays an important role in preclinical research to unravel the precise role of microvascular dysfunction in $\mathrm{HF}$ development [17-20]. The relationship of LV hypertrophy and decline in LV function with regional changes in myocardial perfusion is not well established for this model. One reason might be that quantitative myocardial perfusion measurements in mice are experimentally very challenging. Furthermore, classical microsphere-based perfusion quantification in the mouse heart is invasive and therefore conducted under non-physiological conditions [21, 22]. Also, the latter technique suffers from sampling errors due to the small mouse heart size [23].

Recently, first-pass perfusion MRI protocols have become available enabling non-invasive quantification of mouse heart perfusion values (in $\mathrm{mL} / \mathrm{min} / \mathrm{g}$ ) in vivo [24, 25]. Makowski et al. [25] already showed a significantly decreased perfusion in the mouse infarct area in comparison to other heart wall segments. More recently, we showed that the first-pass perfusion measurements are highly repeatable and that one can determine heart anatomy and function during the same MRI session [26]. This offers the unique opportunity to relate microvascular, functional and hypertrophic adaptations in the same mouse under identical conditions in a longitudinal fashion.

The aim of this study was therefore to apply first-pass perfusion MRI to quantify LV regional myocardial perfusion in a cohort of healthy mice and littermates that underwent TAC resulting in LV pressure overload induced hypertrophy. Perfusion values were related to global LV function and morphology as obtained from cinematographic MRI. Measurements were performed 1, 2, 4, 7 and 10 weeks after TAC.

\section{Methods}

Animal model

A total of 20 C57BL/6 mice ( $\widehat{3}$, age 11 weeks, 24-26 g) were included in this study. Animals were housed under standard laboratory conditions with a $12 \mathrm{~h}$ light/dark cycle and were maintained on a standard diet with access to water ad libitum. All animal experiments were performed according to the Directive 2010/63/EU of the European Parliament and approved by the local Animal Care and Use Committee. Mice were randomly separated in a control group $(n=6)$ and a group that was subjected to TAC $(\mathrm{n}=14)$, resulting in LV pressure overload (see Online Resource 1) [20, 27].

\section{Study protocol}

All MR images were acquired as previously described using a dedicated 9.4T small animal MRI scanner (Bruker BioSpec, Ettlingen, Germany) (see Online Resource 1) [24, 26, 28]. MRI measurements were performed 1, 2, 4, 7 and 10 weeks after surgery. At each session first-pass perfusion images were acquired with a prebolus $\left(25 \mu \mathrm{L}, 40 \mathrm{mM} \mathrm{Gd}(\mathrm{DTPA})^{2-}\right)$ and a full-bolus $\left.(100 \mu \mathrm{L}, 40 \mathrm{mM} \text { Gd(DTPA })^{2-}\right)$. Perfusion quantification was successful in 29/30 measurements in control mice and 59/70 in TAC mice. Missing data points were either due to death of mice in between measurement time points or unsuccessful measurements, primarily due to failed contrast agent infusion. Some datasets were excluded from further analysis as a result of ECG distortions, but only when the distortions led to a severely degraded image quality. In between both boluses a 20 min waiting time ensured sufficient clearance of Gd(DTPA) ${ }^{2-}$ [26]. LV function and mass were quantified during these $20 \mathrm{~min}$ (see Online Resource 1) [28]. Immediately after the last measurements the anesthetized animals were killed by means of perfusing the vascular bed with phosphate buffered saline ( $\mathrm{pH}$ 7.4) infused via a needle penetrating the apex and exsanguination from the vena cava inferior. Next, the integrity of the TAC was visually verified and heart wet weight, lung wet weight ( $\mathrm{LuW})$, and tibia length (TL) were measured.

\section{Data analysis}

Myocardial perfusion ( $\mathrm{mL} / \mathrm{min} / \mathrm{g}$ ) was quantified as described previously (also see Online Resource 1) [26]. Briefly, signal intensity (SI) time curves from the LV blood pool and the LV myocardium were obtained using home-built software in Matlab (The Mathworks, Inc.). We segmented the $\mathrm{LV}$ wall rather conservatively making sure that the regions of interest did only contain pixels well within the myocardial wall ensuring that myocardial signal was not contaminated 
Fig. 1 a Four-chamber longaxis image during diastole overlaid with endocardial and epicardial contours and the five segments used for regional quantification of myocardial perfusion. $S_{\text {base }}$ septum (basal part), $S_{\text {mid }}$ septum (midventricular part), $A$ apex, $L_{\text {base }}$ lateral wall (basal part), $L_{\text {mid }}$ lateral wall (midventricular part). b Corresponding cinematographic diastolic fourchamber long-axis image
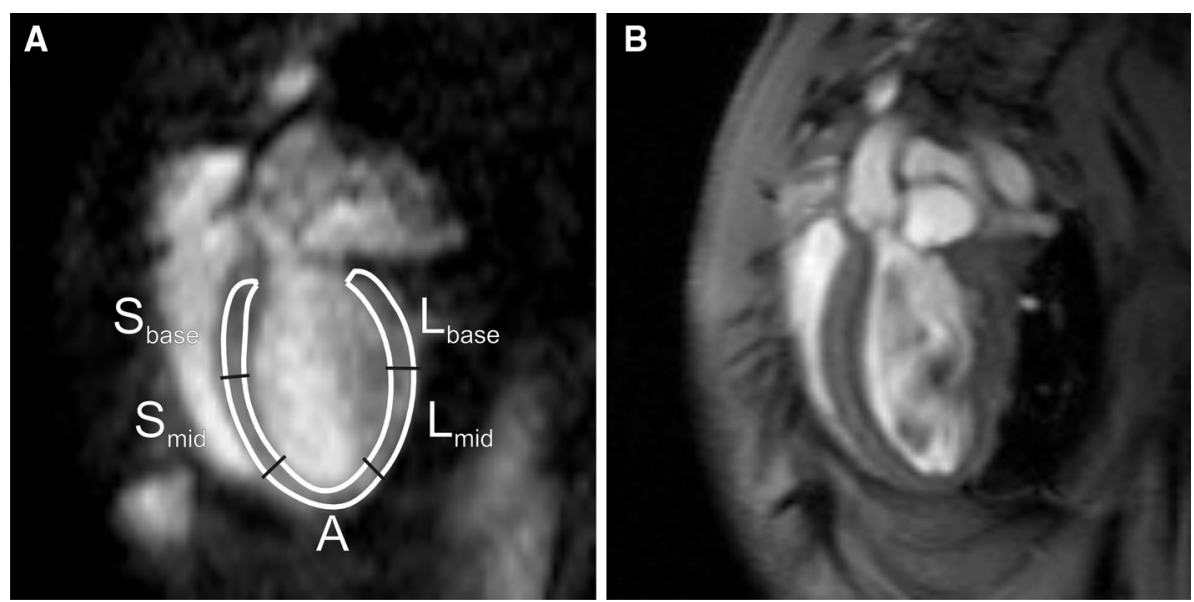

by the LV lumen signal and vice versa. Regions-of-interest were slightly adjusted per frame to correct for small position changes of the heart due to respiration. Pre-injection baseline signal intensity was subtracted from the signal intensity time curve and subsequently the curves were normalized to the pre-injection baseline signal to correct for signal reception inhomogeneities introduced by the RF receive surface coil. For myocardial perfusion quantification, a non-saturated AIF was reconstructed from four time-shifted $\gamma$-variate prebolus fits [29]. Next, perfusion was quantified using a Fermi constrained deconvolution of the myocardial tissue response from the full-bolus with the AIF using a Levenberg-Marquardt least squares fitting algorithm [30]. For regional perfusion analysis the LV myocardium was divided in five segments (Fig. 1). From the $\gamma$-variate fittings also the area under the curve (AUC), and the mean transit time (MTT) defined as the full width at half maximum of the fitted peak, were extracted. The myocardial wall was segmented semi-automatically in the cinematographic MR images using CAAS MRV FARM (Pie Medical Imaging, The Netherlands) to obtain LV function and mass. Whole heart blood flow was calculated by multiplying perfusion with LV mass. Myocardial stroke work was estimated by multiplying stroke volume and mean aortic pressure proximal to the TAC, using $83 \pm 5 \mathrm{mmHg}$ in control and $83 \pm 4 \mathrm{mmHg}$ in TAC mice based on data from van Deel et al. [20]. Finally, the relationship between the cardiac output $(\mathrm{CO})$ and the contrast agent concentration over the first-pass $c_{a}(t)$, as estimated from the AUC of the first-pass in the prebolus injection, was assessed using the Stewart-Hamilton equation $[26,31]$.

$C O=\frac{M}{\int c_{a}(t) d t}$

in which $\mathrm{M}$ is the injected amount of the tracer. Data analysis required approximately one hour for each perfusion measurement.
Statistics

Data are expressed as mean \pm standard deviation (SD). Differences in LV mass/TL, LV EF, stroke volume (SV), cardiac output (CO), heart rate (HR), respiratory rate (Resp) and bodyweight (BW) were tested for statistical significance with an ANOVA for repeated measures with group being control and TAC as factor, followed by the Bonferroni post hoc test when appropriate. In case of interaction between time and group, the effect of time was tested separately per group. Differences in perfusion were tested for statistical significance with an ANOVA for repeated measures with region and group as factor, followed by the Bonferroni post hoc test when appropriate. In TAC mice only linear regression analysis with offset was used to relate myocardial perfusion to LV parameters (EDV, ESV, EF, and LVmass), to relate blood flow to $\mathrm{CO}$, and to stroke work. Changes in LuW/TL, HW/TL, blood flow, and blood flow to the heart as percentage of $\mathrm{CO}$ were tested for statistical significance with one-sided Student's $t$ test. Calculations were performed using SPSS 19.0 (SPSS Inc., Chicago). For all tests the level of significance was set at $\alpha=0.05$.

\section{Results}

All control mice completed the experimental protocol. Two mice died during the course of the experiment at 2 and 7 weeks after TAC, respectively. General animal characteristics are given in Table 1. Mouse bodyweight increased over time $(P<0.001)$, however, no significant differences between groups were detected. Also, no differences in HR or Resp as measured during MRI scanning were detected between control and TAC mice.

LV functional parameters were determined from cinematographic MR images (see Online Resource 1). Impaired 
Table 1 General animal characteristics

\begin{tabular}{llcccrr}
\hline & Week 1 & Week 2 & Week 4 & Week 7 & Week 10 \\
\hline BW $(\mathrm{g})$ & Control & $25.9 \pm 2.0$ & $26.7 \pm 1.6$ & $26.8 \pm 1.4$ & $27.1 \pm 1.5$ & $29.0 \pm 1.7$ \\
& TAC & $24.6 \pm 1.6$ & $25.6 \pm 1.4$ & $26.3 \pm 1.0$ & $26.9 \pm 1.1$ & $27.6 \pm 1.2$ \\
HR $\left(\mathrm{min}^{-1}\right)$ & Control & $515 \pm 43$ & $523 \pm 29$ & $503 \pm 35$ & $550 \pm 31$ & $524 \pm 39$ \\
& TAC & $529 \pm 40$ & $534 \pm 25$ & $545 \pm 23$ & $539 \pm 46$ & $531 \pm 28$ \\
Resp $\left(\mathrm{min}^{-1}\right)$ & Control & $63 \pm 8$ & $48 \pm 4$ & $71 \pm 16$ & $58 \pm 7$ & $62 \pm 11$ \\
& TAC & $58 \pm 9$ & $56 \pm 9$ & $68 \pm 9$ & $69 \pm 12$ \\
\hline
\end{tabular}

Mouse body weight (BW) measured before the MRI measurements, and heart rate (HR) and respiratory rate (Resp) during the MRI measurements at the different time points in the study. For all parameters no significant differences between groups were detected. BW increased over time $(P<0.001)$, HR was constant over time, but Resp at week 2 was somewhat lower as compared to week 4 and $10(P<0.05)$

Table 2 Post-mortem assessment of hypertrophy and pulmonary remodeling

\begin{tabular}{lcl}
\hline Group & Heart mass/TL $(\mathrm{mg} / \mathrm{mm})$ & LuW/TL $(\mathrm{mg} / \mathrm{mm})$ \\
\hline Control & $9.3 \pm 0.9$ & $10.0 \pm 0.9$ \\
TAC & $13.2 \pm 1.8^{*}$ & $13.7 \pm 7.0$ \\
\hline
\end{tabular}

Three TAC mice developed a large lung weight-to-tibia length (LuW/ TL) ratio $(\mathrm{mg} / \mathrm{mm})$ of $18.2,23.3$ and $33.5 \mathrm{mg} / \mathrm{mm}$ as compared to $10.6 \pm 1.7$ for the other mice in the group. This resulted in a slightly elevated group-averaged LuW/TL ratio in the TAC mice, but the differences did not reach significance $(P=0.11)$. Post mortem heart mass normalized to TL was increased in TAC mice as compared to controls $\left({ }^{\ddagger} P<0.001\right)$

systolic function in the TAC mice was apparent from an increased $\operatorname{EDV}(P<0.01)$ and $\operatorname{ESV}(P<0.01)$, and a decreased $\mathrm{EF}(P<0.001)$ as compared to control mice (see Online Resource 1). In TAC mice EDV increased over time from $78 \pm 10 \mu \mathrm{L}$ to $92 \pm 19 \mu \mathrm{L}(P<0.01)$, whereas $\mathrm{ESV}$ $(53 \pm 14 \mu \mathrm{L})$ and $\mathrm{EF}(39 \pm 8 \%)$ remained constant over time. No significant changes over time were detected for $\operatorname{EDV}(70 \pm 11 \mu \mathrm{L}), \operatorname{ESV}(25 \pm 8 \mu \mathrm{L})$ and $\mathrm{EF}(65 \pm 6 \%)$ in the control mice. Myocardial hypertrophy in TAC mice was apparent from an increased LV mass normalized to TL as compared to controls $(P<0.001)$. In control mice $\mathrm{LV}$ mass normalized to TL $(5.3 \pm 0.4 \mathrm{mg} / \mathrm{mm})$ remained constant over time, whereas LV mass in TAC mice gradually increased $(P<0.001)$ from $6.1 \pm 0.8 \mathrm{mg} / \mathrm{mm}$ (week 1$)$ to $7.4 \pm 0.9 \mathrm{mg} / \mathrm{mm}$ (week 10). Moreover, SV was decreased in TAC mice $(33 \pm 7 \mu \mathrm{L})$ as compared to control mice $(45 \pm 7 \mu \mathrm{L})(P<0.001)$, and $\mathrm{CO}$ was also decreased in TAC mice $(17 \pm 3 \mathrm{~mL} / \mathrm{min})$ as compared to controls $(23 \pm 3 \mathrm{~mL} / \mathrm{min})(P<0.001)$. No significant changes over time were detected in both groups for SV and CO.

Post-mortem whole heart weight was increased in TAC mice as compared to controls $(P<0.001)$ (Table 2). LuW/ TL ratio, which was used as a measure for pulmonary remodeling, was only increased in three TAC mice. As a result, the apparent increase of LuW/TL in TAC mice did not reach significance.
Figure 2 shows a series of representative myocardial first-pass perfusion images of a prebolus and full-bolus experiment in a control and TAC mouse. The pictures depict images acquired before and at different time points after injection of a small and a relatively large quantity of Gd(DTPA $)^{2-}$, respectively. Blood and myocardial signals were appropriately nulled before contrast agent injection. For both bolus measurements one can observe the arrival of $\operatorname{Gd}(D T P A)^{2-}$ in the right ventricle, in the left ventricle, and in the myocardium. For the prebolus only minor signal enhancement was observed in the myocardium and signal intensity in the whole image returned quickly to values close to baseline levels, indicating strong dilution and fast clearance of the low volume prebolus.

The mean transit time (MTT) is defined as the average time the bolus requires for passing through a region of interest. MTTs of the LV lumen signal from the prebolus in the control mice $(1.3 \pm 0.5 \mathrm{~s})$ and TAC mice $(1.4 \pm 0.6 \mathrm{~s})$ were not significantly different. Also, the MTT of the LV lumen signal from the full-bolus in the control $(4.8 \pm 0.7 \mathrm{~s})$ and TAC mice $(4.5 \pm 0.6 \mathrm{~s})$ were statistically the same. Figure 3 a displays two examples of arterial input functions (AIF) for a control and a TAC mouse, which served as input for the Fermi analysis to model the measured myocardial tissue response and quantify the perfusion. The TAC mouse AIF was notably higher than that of the control mouse, which was also the case for the group averaged areas under the curve as a measure of the AIF amplitude $(61.9 \pm 32.5$ versus $41.2 \pm 9.9$ for TAC and controls, respectively). The higher AIF was related to a decreased $\mathrm{CO}$ for TAC mice via the Stewart-Hamilton relationship (Eq. 1) (Fig. 3b).

Having established that prebolus and full-bolus injections in both groups resulted in consistent signal-intensity time curves with similar MTTs and AIFs that followed the Stewart-Hamilton relation, myocardial tissue perfusion (in $\mathrm{mL} / \mathrm{min} / \mathrm{g}$ ) was quantified regionally for each mouse. Figure 4 shows fits of the myocardial tissue response curves for two representative cases of a control and a TAC mouse. 


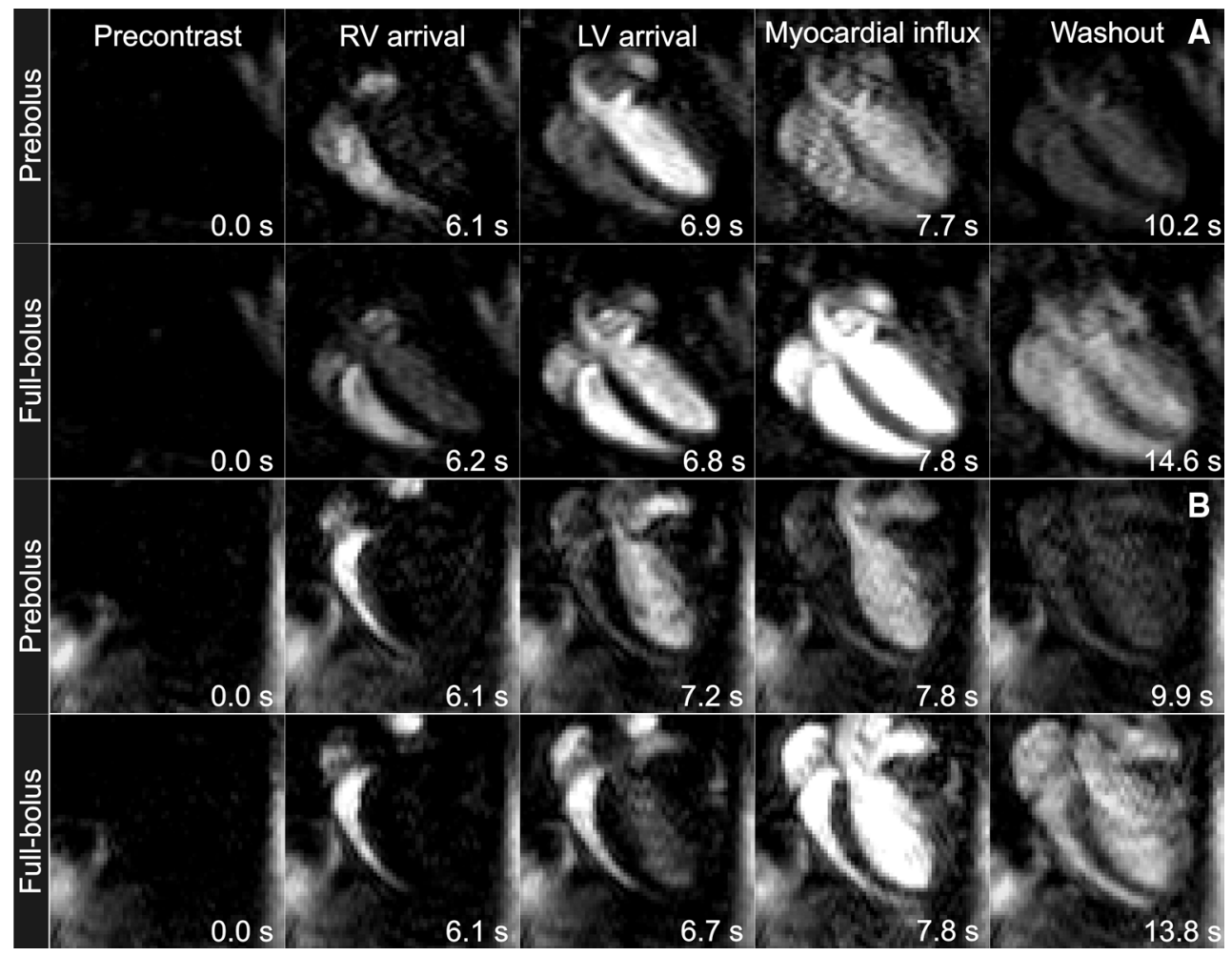

Fig. 2 Time series of cardiac images obtained during the firstpassage of a prebolus $\left(25 \mu \mathrm{L}, 40 \mathrm{mM} \mathrm{Gd}(\mathrm{DTPA})^{2-}\right)$ and a full-bolus $\left(100 \mu \mathrm{L}, 40 \mathrm{mM} \mathrm{Gd}(\mathrm{DTPA})^{2-}\right)$ in a control (two top rows) and TAC mouse (two bottom rows). From left to right: pre-contrast, RV arrival,
LV arrival, myocardial influx, and washout. Indicated are the time points at which each image was acquired with respect to the precontrast image. Examples of cinematographic images of the prebolus and full-bolus passage are given in Online Resource 2
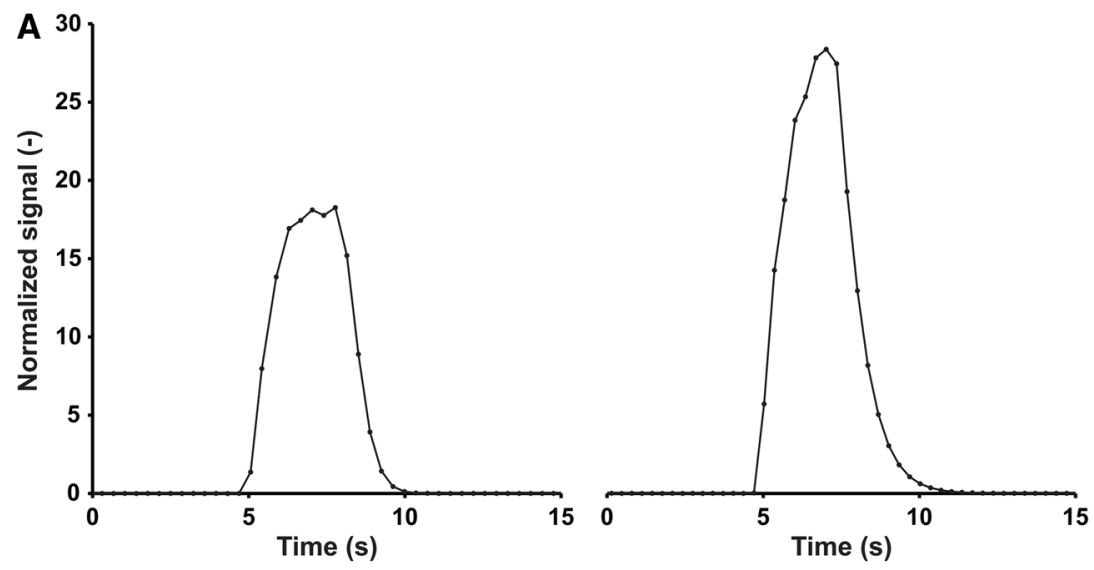

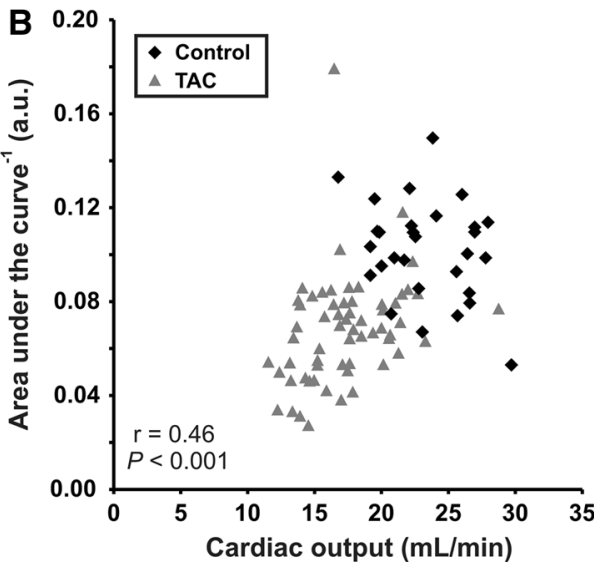

b Relationship between the cardiac output and the reciprocal of the area under the curve (AUC) in control and TAC mice for the prebolus injection. When assuming a linear relationship between both parameters, a moderate relationship was obtained $(\mathrm{r}=0.46, P<0.001)$

the two groups were observed. Unexpectedly, low perfusion values were found in some segments in control mice at week 4 , which we attribute to experimental difficulties at that specific time point (see "Discussion"). Most importantly, myocardial perfusion in TAC mice was consistently

fusion with time were detected for both TAC and control hearts. No regional differences in perfusion values within 
Fig. 4 Measured myocardial tissue responses in the five wall segments and the corresponding modeled signal responses, obtained from the Fermiconstrained deconvolution of the myocardial tissue responses with the AIF in control (top row) and TAC mice (bottom row). $S_{\text {base }}$ septum (basal part), $S_{\text {mid }}$ septum (midventricular part), $A$ apex, $L_{\text {base }}$ lateral wall (basal part), $L_{\text {mid }}$ lateral wall (midventricular part). These segments are depicted in Fig. 1
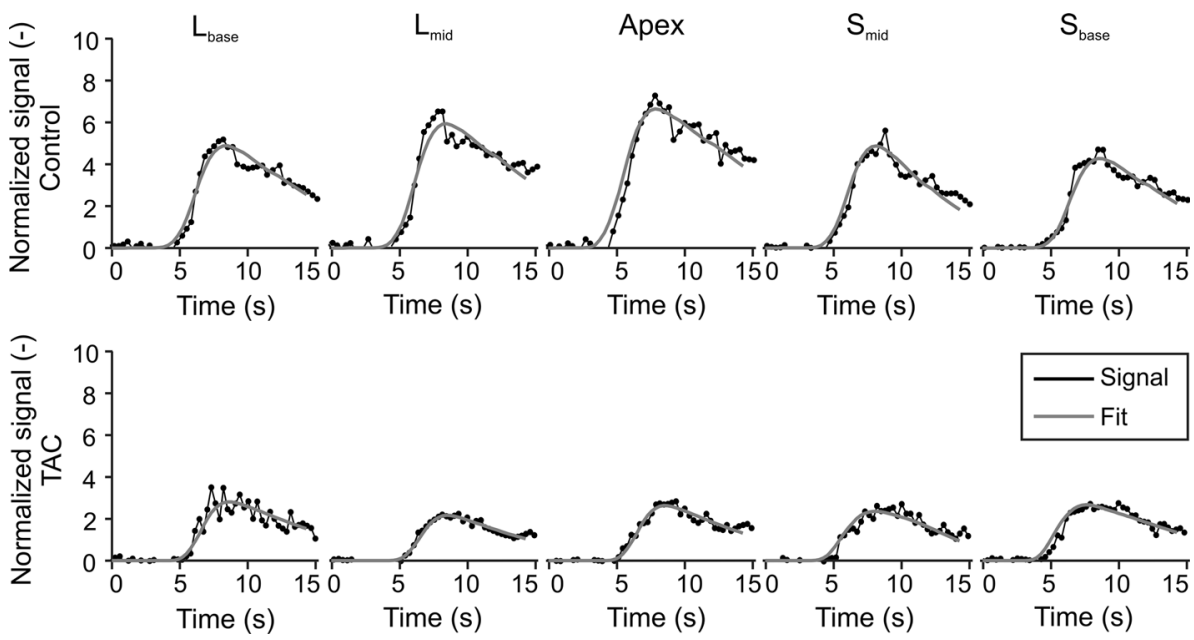
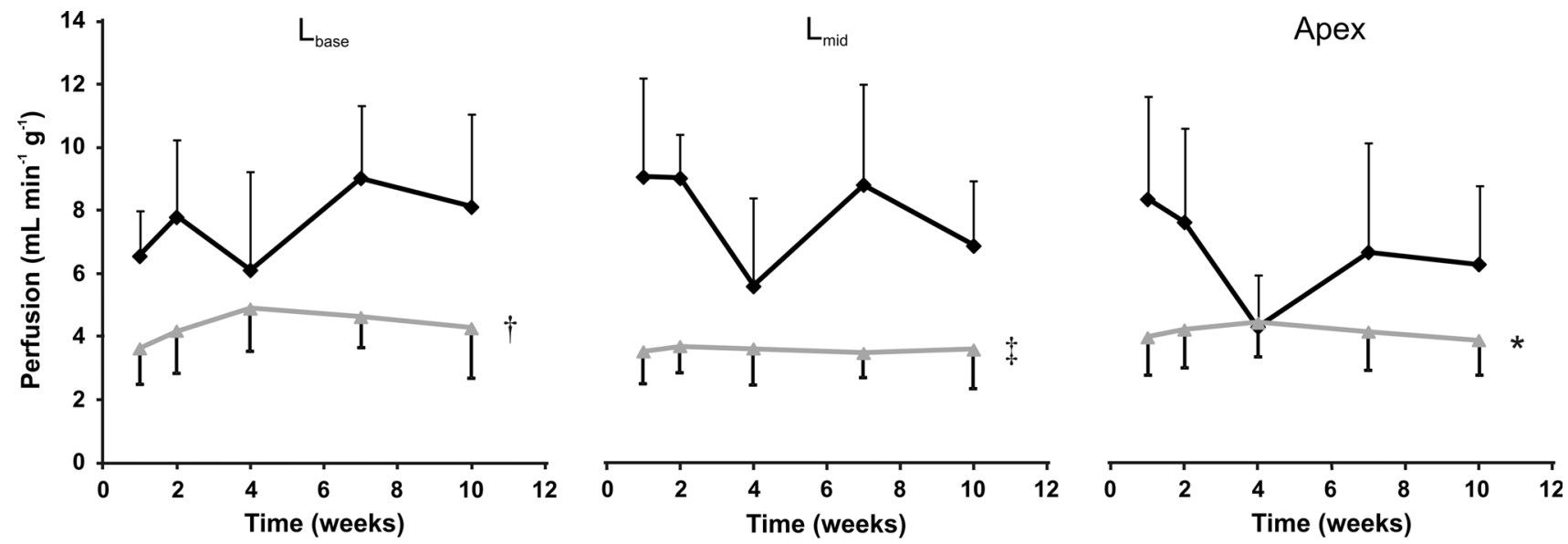

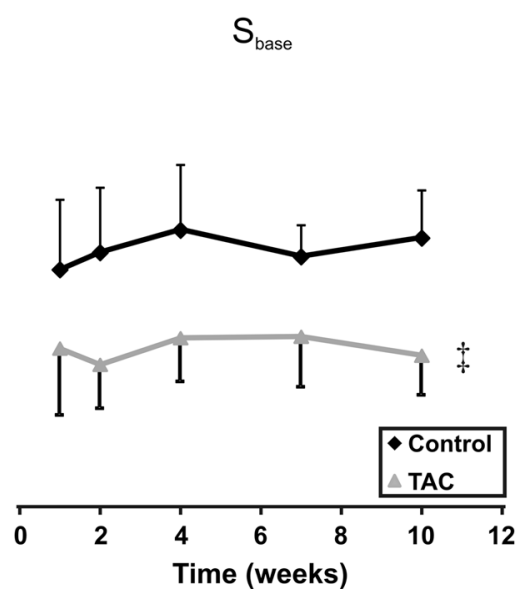

between segments were observed. Low perfusion values were found in the lateral wall and the apex in control mice at week 4, which was attributed to experimental difficulties (see "Discussion"). Mean and SD per time point are denoted by the corresponding symbol and error bars. Statistical differences as compared to the control group are indicated by $* P<0.05,{ }^{\dagger} P<0.01$ and ${ }^{\star} P<0.001$ 

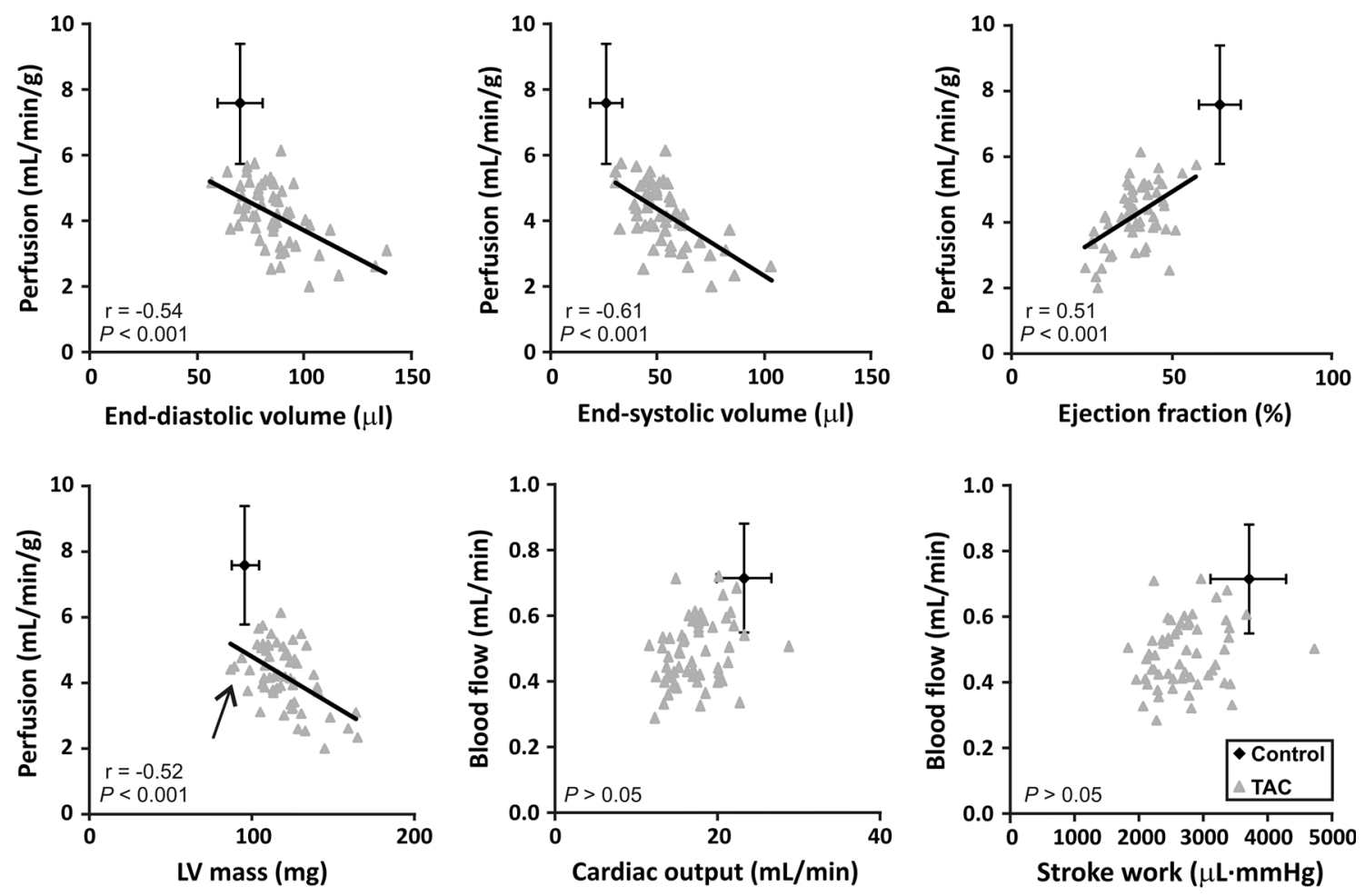

Fig. 6 Relationship between average perfusion $(\mathrm{mL} / \mathrm{min} / \mathrm{g})$ in individual hearts and the left ventricular end-diastolic volume (EDV) (top left), end-systolic volume (ESV) (top middle), ejection fraction (EF) (top right) and LV mass (bottom left), and between the whole heart blood flow and the cardiac output (bottom middle), and the stroke work (bottom right). Whole heart perfusion was calculated by multiplying left ventricular perfusion with LV mass. Stroke work was

lower than in control mice. Region- and time-averaged perfusion in TAC mice was $4.2 \pm 0.9 \mathrm{~mL} / \mathrm{min} / \mathrm{g}$, while $7.6 \pm 1.8 \mathrm{~mL} / \mathrm{min} / \mathrm{g}$ for controls $(P=0.001)$.

In order to examine the relationship between the decreased perfusion to the severity of hypertrophy and decline in function in individual mice, perfusion values of the entire $\mathrm{LV}$ were correlated to several functional parameters (Fig. 6). Decreased myocardial perfusion in TAC mice was correlated to increased LV mass $(\mathrm{r}=-0.52, P<0.001)$, increased $\operatorname{EDV}(\mathrm{r}=-0.54, P<0.001)$, increased ESV $(\mathrm{r}=-0.61$, $P<0.001)$, and decreased EF $(\mathrm{r}=0.51, P<0.001)$. Whole heart blood flow, calculated by multiplying perfusion with LV mass as measure for the total amount of blood supplied to the $\mathrm{LV}$, was decreased in TAC mice $(0.5 \pm 0.1 \mathrm{~mL} / \mathrm{min})$ as compared to controls $(0.7 \pm 0.2 \mathrm{~mL} / \mathrm{min})(P<0.001)$, but whole heart blood flow in TAC mice was not associated with a decreased CO. The reduction in blood flow to the heart expressed as percentage of the $\mathrm{CO}$ in TAC mice $(2.9 \pm 0.7 \%)$ as compared to controls $(3.2 \pm 0.9 \%)$ showed a trend towards significance $(P=0.07)$. Myocardial stroke work, i.e. the work done by the LV to pump a certain amount of blood into the aorta, was estimated by multiplying calculated by multiplying stroke volume with the estimated mean aortic pressure. The mean and SD for the control mice are denoted by the corresponding symbol and error bars. For TAC mice all measurement points are shown and the results (Pearson correlation coefficient and significance) of the linear regression analysis are shown in each panel

stroke volume and mean aortic pressure, as measure for the force delivered by the LV. Stroke work in TAC mice $(2,728 \pm 540 \mu \mathrm{L} \mathrm{mmHg})$ was decreased as compared to control mice $(3,681 \pm 583 \mu \mathrm{L} \mathrm{mmHg}, \quad P<0.001)$, but myocardial stroke work in TAC mice was not correlated to blood flow (Fig. 6).

\section{Discussion}

The TAC mouse is a well-established, valued model for hypertrophy and HF and has been used for studying various aspects of vascular remodeling in myocardial disease [1720, 28]. Despite considerable efforts the precise mechanistic role of decreased myocardial perfusion in the development of HF is not fully elucidated. In particular, the relationship between myocardial perfusion and the evolution and severity of decline in LV function in this mouse model has not been addressed thus far. Here, we therefore investigated the effect of LV pressure overload induced hypertrophy on myocardial perfusion in the TAC mouse model using quantitative first-pass perfusion MRI, and 
related the perfusion data to the severity and time-course of global left ventricular dysfunction.

Hypertrophy in the TAC mice was apparent from a significantly increased LV mass, while considerably increased EDV and ESV denoted LV dilation. Moreover, LV function was compromised, indicated by decreased EF, $\mathrm{SV}$, and CO. Profound pulmonary remodeling, as assessed from the LuW/TL ratio, was only observed in three out of fourteen TAC mice. Taken together, from the anatomical data and MRI measurements it can be concluded that the TAC surgery resulted in a state of stable cardiac dysfunction rather than progressive HF.

Myocardial perfusion was studied under resting conditions, i.e. the vasculature was not maximally vasodilated. Under these conditions, perfusion values in TAC mice were almost twofold lower than those for control mice. Group-averaged perfusion values in both groups were found to be constant over time. The apparent decrease of myocardial perfusion observed at week 4 in the apex and both lateral segments of the control mice was likely due to problems with the cardiac triggering. These occurred in 4 out of the 6 measurements and resulted in severe distortions of the first-pass images. LV mass and EDV in TAC mice progressively increased over time and therefore perfusion values were correlated in more detail to LV function and morphology (Fig. 6).

Decreased myocardial perfusion in TAC mice was clearly correlated to increased EDV and ESV, decreased $\mathrm{EF}$ and increased myocardial mass (Fig. 6). Interestingly, even for the TAC mice without a substantial increase in LV mass, rest perfusion was lower as compared to control mice with the same LV mass (arrow in Fig. 6). Such reduced resting-state perfusion in the absence of LV hypertrophy was also observed in patients with arterial hypertension [32]. We propose that this is the result of a hemodynamic effect caused by the induction of LV pressure overload. The further decrease of perfusion with increased myocardial mass can be attributed to a decreased capillary density, as hypertrophic myocardial growth outweighs neovascularization [33].

Pathological myocardial hypertrophy is associated with an increased cardiac workload causing an increased $\mathrm{O}_{2}$ demand, which should be met by increased myocardial perfusion. Instead, myocardial perfusion and perfusion reserve are impaired in pathological cardiac hypertrophy [10]. The resulting lower $\mathrm{O}_{2}$ delivery capacity has direct functional consequence in terms of impaired myocardial contractility and may even promote apoptosis and fibrosis [7, 34]. In our data we indeed observed a significant impairment of EF simultaneous with an impaired myocardial perfusion and, importantly, a further decrease of perfusion was strongly associated with a further reduction of the EF (Fig. 6).
Myocardial stroke work was estimated by multiplying stroke volume and mean aortic pressure. Invasive pressure measurements, for example with a conductance catheter, could not be combined in the same mice with longitudinal MRI measurements. Therefore, mean aortic pressure in control and TAC mice had to be deduced from earlier observations in slightly older mice and anesthetized using $2.5 \%$ isoflurane in $\mathrm{O}_{2} / \mathrm{N}_{2}(50 / 50 \mathrm{vol} \%)$ [20]. The diminished blood flow to TAC hearts resulted in a decreased stroke work.

In larger mammals perfusion deficits in hypertrophic hearts are often better reflected by impaired perfusion reserve than by resting state perfusion values $[10,35]$. Here, a part of the perfusion reserve is employed to maintain a near normal or even slightly increased baseline perfusion. In contrast, basal metabolism in healthy mice is relatively high resulting in a concurrent high baseline perfusion in mice $(6-8 \mathrm{~mL} / \mathrm{min} / \mathrm{g})$ as compared to larger mammals $(0.5-1.5 \mathrm{~mL} / \mathrm{min} / \mathrm{g})$. This may limit recruitment of perfusion reserve to fully compensate for impaired perfusion in face of LV pressure overload, resulting in detection of perfusion abnormalities under resting conditions in mice. Moreover, even though perfusion values were in agreement with mouse resting state perfusion values previously reported, we cannot exclude that the use of isoflurane as anesthetic might have resulted in some vasodilation and thus in increased resting state myocardial perfusion [36], potentially enhancing perfusion differences between TAC and control mice [37].

Oxygen consumption and therefore myocardial perfusion are closely related to heart rate [38]. For that reason, mouse body temperature and heart rate were carefully kept constant during MRI measurements and between groups. As a result, no relation between heart rate and myocardial perfusion was observed.

Myocardial perfusion measurements were performed in a long-axis orientation to assess possible perfusion differences between basal, midventricular and apical regions [32, 39]. However, such differences were not observed in our data. It remains to be determined whether such differences are manifested when the compensated stage of hypertrophy in the present study progresses to overt HF. The long axis measurements might have resulted in some partial volume effects in slice direction in the presence of the curved myocardial wall, particularly near the apex. However, we found no evidence that partial volume effects interfered with the analysis of regional differences in perfusion values (Fig. 5).

Patients with an aortic valve stenosis often suffer from myocardial ischemia, but the causative mechanisms are poorly understood. Similarly, the relieve of this ischemic condition upon replacement of the aortic valve is also not well understood [40]. First-pass perfusion measurements in 
TAC mice before and after removal of the constriction might help to gain new insights in this phenomenon [41]. Quantification of transmural perfusion, especially when combined with ventricular pressure measurements, could be of particular interest to assess the potential contribution of impaired endocardial perfusion on this ischemic condition.

There are some limitations to this study. First, in future work our method could be extended to measure the perfusion reserve, for example using adenosine or even a higher dose of isoflurane to produce maximum coronary artery vasodilation [10, 42]. This will provide valuable additional information on maximal myocardial perfusion capacity. Hartley et al. [17] studied coronary blood flow velocity using Doppler ultrasound. Coronary flow reserve gradually diminished over time until it was virtually absent, 3 weeks after TAC, mainly as a result of increased baseline coronary flow velocity, also suggesting an impaired myocardial perfusion reserve. In particular, such measurements might be of great value when related to other parameters obtained from a multiparametric MRI protocol. One example is fibrosis, which is known to have detrimental effects on myocardial perfusion [13]. As measurement of the perfusion reserve with the present method requires two dual-bolus injections, the effects of Gd(DTPA $)^{2-}$ accumulation and prolonged anesthesia duration on the repeatability of the perfusion quantification method needs careful consideration. Second, young adult mice were used. Young animals possess the ability to maintain relatively normal capillary density in response to a pressure overload, a capacity that decreases with age [14]. Thus, the use of animals at an early age might lower the relevance for perfusion deficits in HF. Nevertheless, the TAC mice in our study did develop perfusion deficits in response to LV pressure overload.

\section{Conclusions}

Pressure overload induced cardiac hypertrophy in TAC mice significantly reduced myocardial perfusion as compared to their healthy littermates. Moreover, the perfusion deficit in the hypertrophic mouse heart was proportional to $\mathrm{LV}$ volume and mass, and was related to decreased LV ejection fraction. The results from this work show the potential of quantitative first-pass perfusion MRI for the study of the perfusion status of the diseased mouse heart. This technique could also provide valuable information for the quantification of pro-angiogenic therapies for the treatment of ischemic heart disease and to monitor the effects of stem cell therapy, in particular when the perfusion tool is incorporated in a multi-parametric MRI protocol.
Acknowledgments We thank L. Niesen, D. Veraart and J. Habets for biotechnical assistance and T. van Osch (Leiden University Medical Center) for discussions. This research was performed within the framework of the Center for Translational Molecular Medicine, project TRIUMPH (Grant 01C-103), and supported by the Netherlands Heart Foundation.

Conflict of interest None.

\section{References}

1. Denolin H, Kuhn H, Krayenbuehl HP et al (1983) The definition of heart failure. Eur Heart J 4(7):445-448

2. Juenger J, Schellberg D, Kraemer S et al (2002) Health related quality of life in patients with congestive heart failure: comparison with other chronic diseases and relation to functional variables. Heart 87(3):235-241

3. de Couto G, Ouzounian M, Liu PP (2010) Early detection of myocardial dysfunction and heart failure. Nat Rev Cardiol 7(6):334-344

4. Lloyd-Jones D, Adams RJ, Brown TM et al (2010) Heart disease and stroke statistics-2010 update: a report from the American Heart Association. Circulation 121(7):e46-e215

5. McMurray JJ, Stewart S (2000) Epidemiology, aetiology, and prognosis of heart failure. Heart 83(5):596-602

6. Cokkinos DV, Pantos C (2011) Myocardial remodeling, an overview. Heart Fail Rev 16:1-4

7. Frey N, Olson EN (2003) Cardiac hypertrophy: the good, the bad, and the ugly. Annu Rev Physiol 65:45-79

8. Neubauer S (2007) The failing heart-an engine out of fuel. N Engl J Med 356(11):1140-1151

9. Shiojima I, Sato K, Izumiya Y et al (2005) Disruption of coordinated cardiac hypertrophy and angiogenesis contributes to the transition to heart failure. J Clin Invest 115(8):2108-2118

10. Vatner SF, Hittinger L (1993) Coronary vascular mechanisms involved in decompensation from hypertrophy to heart failure. J Am Coll Cardiol 22(4 Suppl A):34A-40A

11. Mathiassen ON, Buus NH, Sihm I et al (2007) Small artery structure is an independent predictor of cardiovascular events in essential hypertension. J Hypertens 25(5):1021-1026

12. Levy BI, Schiffrin EL, Mourad JJ et al (2008) Impaired tissue perfusion: a pathology common to hypertension, obesity, and diabetes mellitus. Circulation 118(9):968-976

13. Dai Z, Aoki T, Fukumoto Y et al (2012) Coronary perivascular fibrosis is associated with impairment of coronary blood flow in patients with non-ischemic heart failure. J Cardiol 60(5):416-421

14. Hoenig MR, Bianchi C, Rosenzweig A et al (2008) The cardiac microvasculature in hypertension, cardiac hypertrophy and diastolic heart failure. Curr Vasc Pharmacol 6(4):292-300

15. Cecchi F, Olivotto I, Gistri R et al (2003) Coronary microvascular dysfunction and prognosis in hypertrophic cardiomyopathy. N Engl J Med 349(11):1027-1035

16. Nakajima H, Onishi K, Kurita $\mathrm{T}$ et al (2010) Hypertension impairs myocardial blood perfusion reserve in subjects without regional myocardial ischemia. Hypertens Res 33(11):1144-1149

17. Hartley CJ, Reddy AK, Madala S et al (2008) Doppler estimation of reduced coronary flow reserve in mice with pressure overload cardiac hypertrophy. Ultrasound Med Biol 34(6):892-901

18. Givvimani S, Munjal C, Gargoum R et al (2011) Hydrogen sulfide mitigates transition from compensatory hypertrophy to heart failure. J Appl Physiol 110(4):1093-1100

19. Oudit GY, Kassiri Z, Zhou J et al (2008) Loss of PTEN attenuates the development of pathological hypertrophy and heart failure in response to biomechanical stress. Cardiovasc Res 78(3):505-514 
20. van Deel ED, de Boer M, Kuster DW et al (2011) Exercise training does not improve cardiac function in compensated or decompensated left ventricular hypertrophy induced by aortic stenosis. J Mol Cell Cardiol 50(6):1017-1025

21. Streif JUG, Nahrendorf M, Hiller K-H et al (2005) In vivo assessment of absolute perfusion and intracapillary blood volume in the murine myocardium by spin labeling magnetic resonance imaging. Magn Reson Med 53(3):584-592

22. Jacquier A, Kober F, Bun S et al (2011) Quantification of myocardial blood flow and flow reserve in rats using arterial spin labeling MRI: comparison with a fluorescent microsphere technique. NMR Biomed 24(9):1047-1053

23. Decking UKM, Pai VM, Bennett E et al (2004) High-resolution imaging reveals a limit in spatial resolution of blood flow measurements by microspheres. Am J Physiol Heart Circ Physiol 287(3):H1132-H1140

24. Coolen BF, Moonen RPM, Paulis LEM et al (2010) Mouse myocardial first-pass perfusion MR imaging. Magn Reson Med 64(6):1658-1663

25. Makowski M, Jansen C, Webb I et al (2010) First-pass contrastenhanced myocardial perfusion MRI in mice on a 3-T clinical MR scanner. Magn Reson Med 64(6):1592-1598

26. van Nierop BJ, Coolen BF, Dijk WJR et al (2012) Quantitative first-pass perfusion MRI of the mouse myocardium. Magn Reson Med 69(6):1735-1744

27. Rockman HA, Ross RS, Harris AN et al (1991) Segregation of atrial-specific and inducible expression of an atrial natriuretic factor transgene in an in vivo murine model of cardiac hypertrophy. Proc Natl Acad Sci USA 88(18):8277-8281

28. van Nierop BJ, van Assen H, van Deel ED et al (2013) Phenotyping of left and right ventricular function in mouse models of compensated hypertrophy and heart failure with cardiac MRI. PLoS One 8(2):e55424

29. Köstler H, Ritter C, Lipp M et al (2004) Prebolus quantitative MR heart perfusion imaging. Magn Reson Med 52(2):296-299

30. Jerosch-Herold M, Wilke N, Stillman AE (1998) Magnetic resonance quantification of the myocardial perfusion reserve with a Fermi function model for constrained deconvolution. Med Phys 25(1):73-84
31. Valentinuzzi ME, Geddes LA, Baker LE (1969) A simple mathematical derivation of the Stewart-Hamilton formula for the determination of cardiac output. Med Biol Eng 7(3):277-282

32. Kawecka-Jaszcz K, Czarnecka D, Olszanecka A et al (2008) Myocardial perfusion in hypertensive patients with normal coronary angiograms. J Hypertens 26(8):1686-1694

33. Izumiya Y, Shiojima I, Sato K et al (2006) Vascular endothelial growth factor blockade promotes the transition from compensatory cardiac hypertrophy to failure in response to pressure overload. Hypertension 47(5):887-893

34. Duncker DJ, de Beer VJ, Merkus D (2008) Alterations in vasomotor control of coronary resistance vessels in remodelled myocardium of swine with a recent myocardial infarction. Med Biol Eng Comput 46(5):485-497

35. Bache RJ (1988) Effects of hypertrophy on the coronary circulation. Prog Cardiovasc Dis 30(6):403-440

36. Kober F, Iltis I, Cozzone PJ et al (2004) Cine-MRI assessment of cardiac function in mice anesthetized with ketamine/xylazine and isoflurane. Magn Reson Mater Phys Biol Med 17(3-6):157-161

37. You J, Wu J, Ge J et al (2012) Comparison between adenosine and isoflurane for assessing the coronary flow reserve in mouse models of left ventricular pressure and volume overload. Am J Physiol Heart Circ Physiol 303(10):H1199-H1207

38. Braunwald E (1971) Control of myocardial oxygen consumption: physiologic and clinical considerations. Am J Cardiol 27(4):416-432

39. Soler R, Rodríguez E, Monserrat L et al (2006) Magnetic resonance imaging of delayed enhancement in hypertrophic cardiomyopathy: relationship with left ventricular perfusion and contractile function. J Comput Assist Tomogr 30(3):412-420

40. Gould KL, Carabello BA (2003) Why angina in aortic stenosis with normal coronary arteriograms? Circulation 107(25):3121-3123

41. Gao X-M, Kiriazis H, Moore X-L et al (2005) Regression of pressure overload-induced left ventricular hypertrophy in mice. Am J Physiol Heart Circ Physiol 288(6):H2702-H2707

42. Kober F, Iltis I, Cozzone PJ et al (2005) Myocardial blood flow mapping in mice using high-resolution spin labeling magnetic resonance imaging: influence of ketamine/xylazine and isoflurane anesthesia. Magn Reson Med 53(3):601-606 